\title{
COMPOSICIÓN NUTRICIONAL DE FRUTOS DE SYAGRUS ROMANZOFFIANA (PINDÓ) NATIVOS DE FORMOSA- ARGENTINA
}

KARINA MARIELA ROMPATO ${ }^{*}$ RODRIGO RUBÉN FRANCO ${ }^{2 *}$ SUSANA NÉLIDA SOMOZA ${ }^{3^{* * *}}$ LUIS SANTIAGO ROMPATO ${ }^{* * * * *}$

\begin{abstract}
La palmera "pindó" (Syagrus romanzoffiana) es una especie nativa, abundante en la provincia de Formosa, Argentina. Las plantas producen frutos con formas diferentes, ovalados y esféricos. Se determinó el perfil de ácidos grasos (AG) en los frutos enteros y antioxidantes y proteínas en pulpa seca molida. Se indagó si las diferentes formas implican variación en la composición nutricional y en antioxidantes. Los datos experimentales arrojaron diferencias en el perfil de ácidos grasos según la forma. Los frutos esféricos, muestran una relación entre ácidos grasos, saturados e insaturados, beneficiosa para la salud humana, con un perfil intermedio entre el aceite de oliva y girasol. Las proteínas en frutos ovalados fue de $3,57 \mathrm{~g} / 100$ $\mathrm{g}$ y $3,66 \mathrm{~g} / 100 \mathrm{~g}$ en esféricos. Entre los antioxidantes, la vitamina $\mathrm{C}$ presenta valores con diferencia significativa entre los frutos ovalados y esféricos, conteniendo estos últimos mayor concentración, $5,23 \mathrm{mg} / \mathrm{g}$. Se encontraron diferencias significativas en compuestos fenólicos según la forma de los frutos, 7,53 mgEAG/100 g para los esféricos. La determinación de carotenoides totales fue de 160,64 en ovalados y $213,56 \mu \mathrm{g} \beta$-caroteno/g en esféricos. El perfil de AG de frutos esféricos y la proporción de aceites encontrada, los convierte en un producto apto para la extracción de aceites comestibles beneficioso para la salud dada su proporción de AG saturados/insaturados, $(1 / 2,5)$ utilizando el fruto entero. El alto contenido de compuestos bioactivos, polifenoles, carotenoides y vitamina $C$ realza la calidad nutricional de la pulpa de éstos frutos y su potencial uso para enriquecimiento de diferentes productos alimenticios.
\end{abstract}

PALABRAS CLAVE: PINDÓ, PALMERA, COMPOSICIÓN NUTRICIONAL, ANTIOXIDANTES, ACEITE.

\footnotetext{
* $\quad$ Bioquímica. Laboratorio de Investigación en Alimentos. Facultad de Ciencias de la Salud. Universidad Nacional de Formosa (UNaF). Formosa. Argentina. Correo electrónico: labalimentos@fs.unf.edu.ar

** Licenciado en Bromatología. Laboratorio de Investigación en Alimentos. Facultad de Ciencias de la Salud. UNaF. Correo electrónico: franco_88@hotmail.com

*** Magister en Salud Pública. Laboratorio de Investigación en Alimentos. Facultad de Ciencias de la Salud. UNaF. Correo electrónico: catsaludpublica@fs.unf.edu.ar

**** Ingeniero Químico. Laboratorio de Investigación en Alimentos. Facultad de Ciencias de la Salud. UNaF. Correo electrónico: labalimentos@fs.unf.edu.ar
} 


\section{INTRODUCCIÓN}

En Formosa crece en forma natural y abundante la palmera "pindó" (Syagrus romanzoffiana), su dispersión comprende el centro este y sur de Brasil, Paraguay, Bolivia, Uruguay y el NEA (Nor Este Argentino, incluida las porciones orientales de Santa Fe, Chaco y Formosa) (CABRAL \& CASTRO, 2007). En general se la utiliza con fines ornamentales en avenidas, jardines y plazas.

Su fruto es drupáceo, globoso-aovado, amarillo anaranjado, cuyo aspecto recuerda a un dátil, de unos $25 \mathrm{~mm}$ de largo y $15 \mathrm{~mm}$ de diámetro, que aloja una sola semilla, aovada, apiculada en ambos extremos. La pulpa poco abundante, es dulce, fibrosa y algo gomosa, comestible, de sabor agradable (LÓPEZ et al., 1987; CROCOMO \& MELO, 1996).

Bonomo y Capeletti (2014) describen los usos étnicos de la palmera pindó relacionándolos principalmente con la subsistencia, la manufactura de artefactos y la construcción de viviendas. Los hetá de la cuenca del Alto Paraná consumen los cogollos de diversas maneras: crudos (palmitos), transformados y almacenados como harina (incluido el tronco), asados, fritos o hervidos en sopa o con miel; el fruto es rico en aceites y proteínas y se emplea en bebidas fermentadas ( $p$. ej. mbochochó), la semilla, se utiliza como alimento fresco; también se consumen las larvas que se crían en los troncos derrumbados (BONOMO \& CAPELETTI, 2014). El fruto es apreciado por una diversidad de aves y también por algunas personas.. El pindó, y la butia (Butia yatay) son los frutos de palma más utilizados en la pesca deportiva del pacú (Piaractus mesopotamicus) en ríos y riachos de la zona. La población criolla, consume el fruto como tal, y suelen utilizarlo en la elaboración de licores, debido a su distinguido aroma y sabor.

Se trata de una especie poco estudiada en nuestro país desde el aspecto nutricional. La mayoría de los datos han sido aportados por investigadores brasileros. Coimbra y Jorge (2011) han publicado la composición proximal del fruto, semilla y pulpa, destacando su contenido en fibras y en compuestos bioactivos como carotenoides y tocoferoles, convirtiéndolo en un producto apto para la elaboración de panificados por ejemplo (COIMBRA \& JORGE, 2011).

Los mismos autores refieren que los contenidos de lípidos es diferente entre la semilla y la pulpa, sugiriendo que los aceites de semilla, en su mayoría saturados, podrían ser útiles en las industrias cosmética y farmacéutica, a diferencia de la pulpa, que además de contener ácidos grasos insaturados, ácido oleico y ácido linoleico, es rica en carotenoides y tocoferoles (COIMBRA \& JORGE, 2012).

Por otro lado se ha observado que los galactomananoglucanos de la pulpa de pindó presentaron funcionalidad antiinflamatoria puesta de manifiesto en ratones con edemas inducidos (PEREIRA DA SILVA \& PAZ PARENTE, 2010).

También se han aislado estilbenoides con propiedad inhibitoria de a-glucosidasas y por lo tanto hipoglucemiantes, del extracto etanólico de semillas de pindó (LAM et al., 2008).

En la naturaleza existen muchas sustancias con propiedades antioxidantes, la gran mayoría son de origen vegetal y están ampliamente difundidas en los alimentos por lo que son incorporados en nuestro organismo a través de la dieta. Numerosos estudios indican que el consumo de frutas y verduras está relacionado con una menor incidencia de enfermedades cardiovasculares y algunos tipos de cáncer, sugiriendo que una dieta rica en estos alimentos ejerce efectos benéficos en la salud debido a la acción de sus componentes antioxidantes (NAZARENO et al, 2008).

Los antioxidantes han adquirido gran relevancia en los últimos años. Actualmente estas sustancias están siendo estudiadas en los laboratorios de investigación de la industria alimentaria, debido a su impacto en la mejora de los alimentos y consecuentemente en la salud humana (PALENCIA MENDOZA, 1999)

Con respecto a su clasificación es posible distinguir los de origen endógeno, biosintetizados por el organismo y los que ingresan a través de la dieta. Estos son: las vitaminas antioxidantes, que comprenden al ácido ascórbico (o Vitamina C); la Vitamina E, y los compuestos Pro-Vitamina A (representados por el beta-caroteno, el alfa-caroteno y la beta- criptoxantina); los carotenoides, 
entre los cuales destacan la luteína, el licopeno, la zeaxantina y la astaxantina, y los polifenoles, caracterizados como compuestos flavonoides (más abundantes en la naturaleza) y los no-flavonoides, en su mayoría alcoholes, ácidos fenólicos y estilbenos (NDHLALA, MOYO, \& VAN STADEN, 2010).

Debido a la importancia de estos componentes bioactivos en la salud, tanto humana como animal (GUTIÉRREZ, ORTIZ, \& CISNEROS, 2008) se hace casi imprescindible incorporar su determinación en la evaluación nutricional de los frutos de las palmas en estudio, además de los macro y micronutrientes.

En la provincia de Formosa, las plantas de pindó, tienen frutos con formas diferentes, algunas producen frutos de forma ovalada, o aovada, y otras globosos, casi esféricos. Bonano y Capeletti (2014) también refieren, en cierta manera, a la forma de los pindó, diciendo que la morfología externa de los pindó colectados tiene, forma aovada, $97 \%$ y el $3 \%$ subglobosa. Otros autores han observado diferencias en la actividad antioxidante de los cultivos por efectos de factores climáticos, prácticas agrícolas, temporada de cosecha, estado de madurez y tipo de almacenamiento entre otros (NAZARENO, 2008)

Este trabajo tuvo como objetivo general analizar y comparar la composición nutricional y en antioxidantes de los frutos de las palmas Syagrus romanzoffiana nativas de la provincia de Formosa, teniendo en cuenta, la ubicación geográfica de las plantas, y las diferentes formas de los frutos.

La información obtenida podrá ser incoporada a las tablas de composición nutricional y se podrá comparar con los datos publicados sobre este tipo de alimento de otra procedencia

\section{MATERIALES Y METODOS}

El trabajo se dividió en dos etapas, en la primera se investigó la influencia de factores ambientales (ubicación geográfica), y fenotípicos (forma de los frutos) en la composición nutricional de los frutos enteros, secados y molidos.

En la segunda, se determinó el perfil nutricional (proteínas y antioxidantes), de la pulpa de los frutos secada y molida, teniendo en cuenta su forma.

\subsection{SELECCIÓN Y RECOLECCIÓN DE MUESTRAS}

\subsubsection{Primera Etapa}

Se recolectaron frutos maduros de palmeras pindó en la localidad de Bella Vista (Dpto Pilagás, Formosa) y en la ciudad de Formosa. Se estableció como criterio de madurez de los frutos, el color naranja oscuro, solo se procesaron los frutos que no presentaron abolladuras, ni signos de deterioro, con la piel intacta. Se lavaron cuidadosamente con agua corriente y se secaron. A fin de determinar las características físicas de los frutos se tomaron 10 frutos al azar de manera de tener representados todos los tamaños y se los pesó. Posteriormente separó la pulpa de la semilla para determinar la proporción de ambos componentes. Los frutos fueron secados en estufa a $70^{\circ} \mathrm{C}$ y se molieron enteros en molino a martillo. Se los almacenó en bolsas con la identificación de la ubicación del árbol de donde se obtuvieron y de la forma de los frutos.

\subsubsection{Segunda Etapa}

Se recolectaron frutos maduros de palmeras pindó ubicadas en el campus universitario y alrededores, $1 \mathrm{Kg}$ de frutos de cada tipo provenientes de 5 palmeras con frutos ovalados y de 5 palmeras con frutos esféricos. Se estableció como criterio de madurez de los frutos, el color naranja oscuro. Se recogieron directamente de la tierra, aquellos que estaban firmes y que no presentaban lesiones. Inmediatamente, estos frutos enteros, fueron lavados, secados y etiquetados según su forma y luego almacenados a $-20^{\circ} \mathrm{C}$ para su conservación, hasta su análisis. 
Al momento de emplearlos se tomaron a la suerte varios frutos de cada árbol y se atemperaron para su procesamiento. Para ello se extrajeron manualmente pulpa y cáscara, con ayuda de un cuchillo, posteriormente se acondicionaron en secador a aire caliente a $50{ }^{\circ} \mathrm{C}$ para secarlas hasta peso constante.

El secado permitió molerlas en un molino a hélice y obtener así la harina, que posteriormente fue almacenada en envase de vidrio a temperatura de refrigeración.

\subsection{MÉTODOS}

Para determinar humedad por gravimetría, los frutos enteros, se secaron en estufa a $105^{\circ} \mathrm{C}$, según procedimiento descripto por AOAC 925.40 (AOAC, 2005); las cenizas por incineración en mufla según procedimiento de la AOAC 950.49 (AOAC, 2005); la proteína bruta de determinó por el método de Kjeldahl descripto por AOAC 920.152 (AOAC, 2005) se determinó amonio por el método de Nessler, previa confección de la curva estandar con solución de $\mathrm{NH}_{4} \mathrm{Cl}$. Los lípidos totales se determinaron por el método de Soxhlet, AOAC 920.39 (AOAC, 2005), modificado. Los hidratos de carbono, tanto azúcares reductores como totales, se determinaron por el método de Fehling según AOAC 974.06 (AOAC, 2005), con curva estandar realizada con glucosa.

El perfil lipídico se realizó por cromatografía gaseosa: la extracción y purificación de la grasa para la cuantificación de los ácidos grasos se realizó de acuerdo al método de Bligh y Dyer (1959), usando BHA en una concentración de 0,01\% como antioxidante. La determinación de los ácidos grasos saturados, monoinsaturados, poliinsaturados y ácidos grasos trans, los metilésteres se realizó de acuerdo con la Técnica de la AOAC 969.33. Esta determinación se efectuó en la Facultad de Agroindustrias de la UNCAus.

Determinación de polifenoles. Se pesó $1 \mathrm{~g}$ de la harina de cada una de las 10 muestras, se las diluyó en $9 \mathrm{ml}$ de agua destilada y homogeneizaron. Se dejó reposar por 60 minutos y luego se filtraron, y el producto de la filtración se utilizó para la determinación de polifenoles con la técnica de Folin-Ciocalteu según Palomino et al. (2009). Se usaron soluciones de ácido gálico (Biopack®) entre $50-500 \mu \mathrm{g} / \mathrm{ml}$ para construir la curva de calibración y los resultados se expresaron en $\mathrm{mg}$ equivalentes de ácido gálico por $100 \mathrm{~g}$ de muestra seca (mgEAG/100 g).

Determinación de ácido ascórbico. La concentración de ácido ascórbico fue determinada por el método de titulación usando el 2,6-dicloroindofenol (AOAC, 2005). Se preparó una solución estándar de ácido ascórbico $(1 \mathrm{mg} / \mathrm{ml})$. Los resultados se expresaron en $\mathrm{mg}$ de ácido ascórbico por gramo de muestra seca $(\mathrm{mgAA} / \mathrm{g})$.

Determinación de Carotenoides totales. Para extracción de los carotenoides se siguió la metodología propuesta por Rodriguez-Amaya (2001). Se midió la absorbancia a la longitud de onda de máxima absorción encontrada $(443 \mathrm{~nm})$. La cantidad de carotenoides totales presentes en la muestra se calculó por medio de la curva estándar y fue expresada como $\mu \mathrm{g}$ de beta-carotenos por $\mathrm{g}$ de muestra seca.

Determinación de proteínas totales. Se realizó según el método descripto por la AOAC 920.152 (AOAC, 2005), con algunos cambios. Se determinó concentración de amonio por el método de Nessler, peviamente se realizó una curva de calibrado (por triplicado) utilizando una solución de $\mathrm{NH}_{4} \mathrm{Cl} 3,82 \mathrm{~g} / \mathrm{L} 100$ veces diluida.

\subsection{TRATAMIENTO ESTADÍSTICOS}

Para realizar el análisis estadístico se evaluaron las 10 muestras obtenidas en la segunda etapa, 5 de cada tipo, y se realizó cada determinación por triplicado. Los datos se procesaron estadísticamete con el programa InfoStat ${ }^{\circledR}$ se realizó el análisis de la varianza y la prueba de diferencias de media de Tukey $(\alpha=0,05)$. 


\section{RESULTADOS Y DISCUSIÓN}

\subsection{PRIMERA ETAPA}

Los pesos de los frutos estuvieron entre 3,53 y $6,26 \mathrm{~g}$ con una media de $5,20 \mathrm{~g}$, correspondiendo un $66,8 \%$ del peso a la pulpa y un $33,2 \%$ a la semilla. Respecto a los pesos de los frutos analizados, son muy similares a los evaluados por Bonano y Capeletti (2014) que con 176 frutos obtuvieron $1000 \mathrm{~g}$, lo que significa una media de 5,68 g por cada fruto, además determinaron un $64,8 \%$ de pulpa y la suma de endocarpio y semilla $35,2 \%$. Goudel (2012) determinó una menor proporción de pulpa 59,29\% y un peso similar, con una media de 5,61 g(GOUDEL, 2012).

Se determinó que la humedad de los frutos enteros varía entre 31 y $59 \mathrm{~g} / 100 \mathrm{~g}$ y las cenizas de 1,38 a $1,53 \mathrm{~g} / 100 \mathrm{~g}$ con una media de $1,45 \mathrm{~g} / 100 \mathrm{~g}$. Los azúcares reductores se determinaron en un rango entre $5,7 \mathrm{~g} / 100 \mathrm{~g}$ a $6,3 \mathrm{~g} / 100 \mathrm{~g}$ y los totales de $7,57 \mathrm{~g} / 100 \mathrm{~g}$ al 8,47 $\mathrm{g} / 100 \mathrm{~g}$ en frutos enteros.

La proporción de proteínas se estableció entre 1,70 y $3,7 \mathrm{~g} / 100 \mathrm{~g}$.

Con respecto a los lípidos, los frutos de pindó secos enteros molidos presentaron una media de 13,34 g/100 g en materia seca. Los datos de lípidos obtenidos no pueden compararse con los de otros autores porque la mayoría los ha determinado separando la pulpa del carozo, de todas maneras si consideramos el trabajo de Coimbra y Jorge (2011), relacionando la cantidad de lípidos que encontraron en carozo, $46 \mathrm{~g} / 100 \mathrm{~g}$, en la pulpa, $7,5 \mathrm{~g} / 100 \mathrm{~g}$ y teniendo en cuenta que la proporción de la pulpa es $65 \%$ del peso del fruto, y el carozo $35 \%$, surge del cálculo una estimación total de 21,0 $\mathrm{g} / 100 \mathrm{~g}$ de lípidos, valor superior al determinado en este trabajo. Estos autores determinaron un valor mucho mayor de lípidos en pulpa, 7,5 g/100 g, que los hallados por Goudel (2012) 2,42 g/100 g, pero menor que los obtenidos por Guimarães (2013), $11 \mathrm{~g} / 100 \mathrm{~g}$ en harina de pulpa de pindó.

Como se muestra en el Gráfico 1, el perfil de ácidos grasos en los frutos estudiados, presenta diferencias debido a la forma de los frutos y no a la localización del árbol. El ácido graso mas abundante en ambas formas es el ácido oleico (18:1 n9 cis) aunque la proporción es diferente variando, entre el 23 (ovalados) al 50\% (esféricos). El ácido láurico (12:0) con una proporción entre 22 y $24 \%$ (ovalados) o el ácido linoleico (18:2 n6 cis) en 17\% (esféricos), aparecen en segundo lugar según la forma del fruto. La concentración del ácido palmítico (16:0) varía entre el 13 al 18\%, según la forma del fruto.

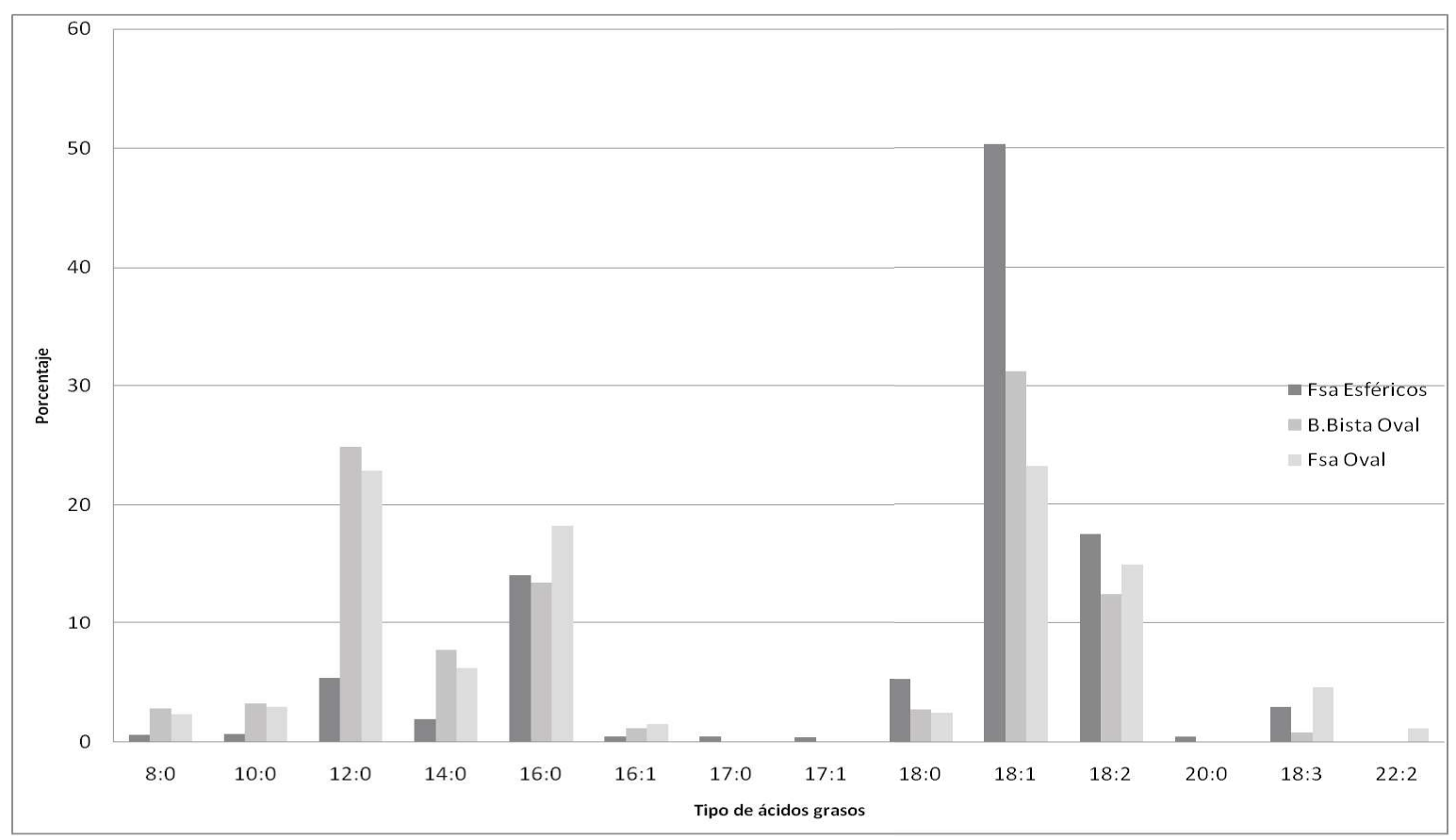

GRÁFICO 1._COMPARACIÓN DE LA COMPOSICIÓN LIPÍDICA DE LOS FRUTOS DE PINDÓ SEGÚN ORIGEN Y FORMA DE LOS FRUTOS ESTUDIADOS. 
Los resultados muestran diferencias en la proporción de ácidos grasos (AG) según la forma de los frutos. Los frutos de forma esférica presentan un predominio de ácido oleico (18:1 n9) de un $50 \%$, y un total de $70 \%$ de ácidos grasos insaturados, que los convierte en una potencial fuente de extracción de aceite comestible. En cambio los frutos de forma ovoide, presentan un $25 \%$ aproximadamente de ácido oleico con un predominio de ácidos grasos saturados de cadena corta. Analizando los datos publicados por Coimbra y Jorge (2011) se encuentran semejanzas con el perfil de ácidos grasos obtenidos para los frutos ovalados. La relación AG saturados/insaturados resultante del perfil de pindó esférico es $1 / 2,5$ y la de los ovalados es $1 / 0,80$, la determinada por Coimbra y Jorge (2011) para pulpa es de $1 / 1,48$ y semilla $1 / 0,47$.

El perfil de AG de pindó esféricos tiene una composición casi intermedia entre el de aceite de oliva y el de girasol, el Gráfico 2 permite visualizar con mayor detalle esta comparación.

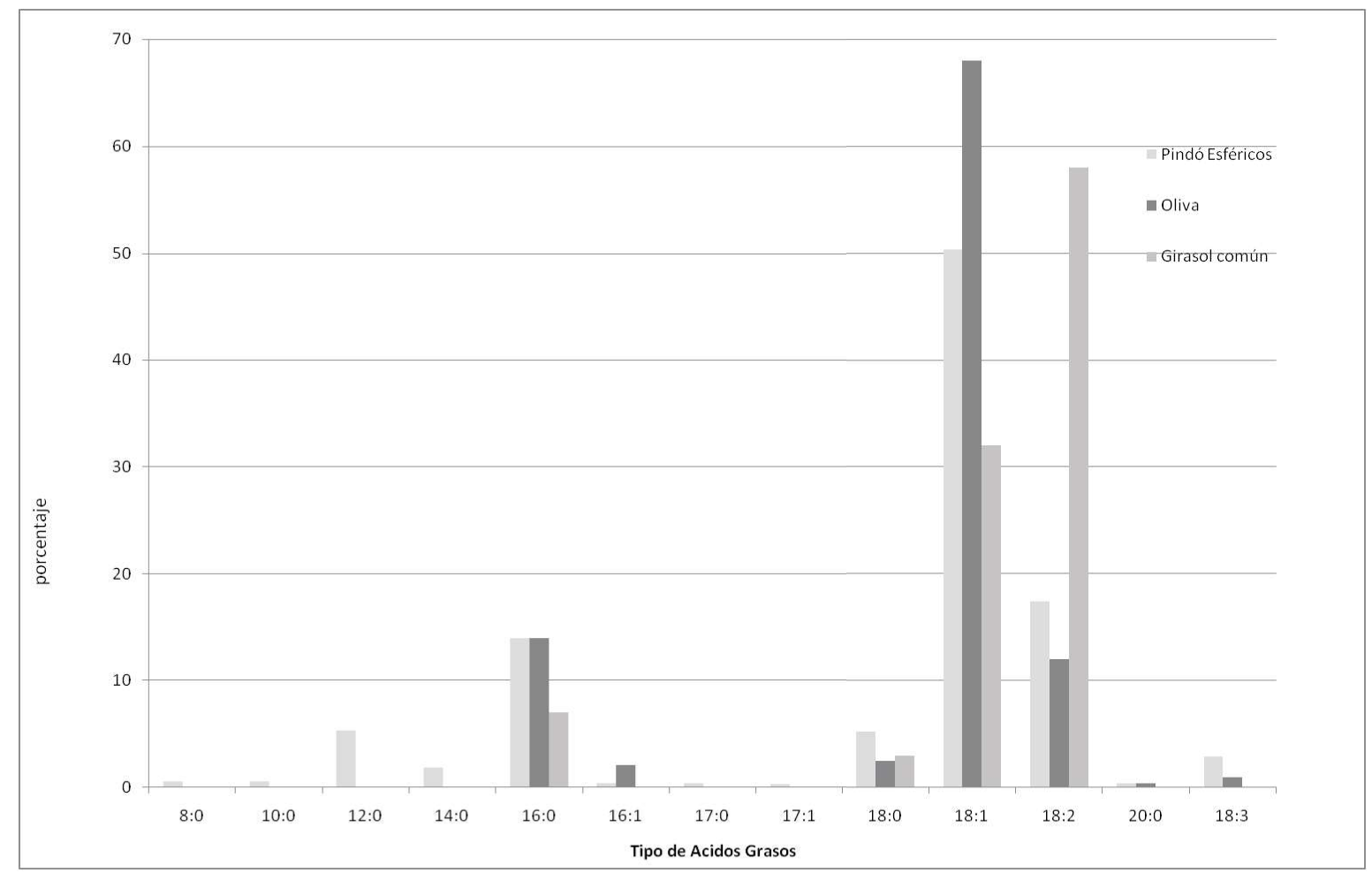

\section{GRÁFICO 2: COMPARACIÓN DE LOS PERFILES DE ÁCIDOS GRASOS DE FRUTOS DE PINDÓ ESFÉRICOS CON ACEITES DE OLIVA Y GIRASOL, SEGÚN DATOS EXPERIMENTALES Y VALORES MEDIOS DEL CÓDIGO ALIMENTARIO ARGENTINO.}

El aceite extraído de los frutos esféricos de pindó resulta en un recurso potencialmente aprovechable con fines alimenticios debido a la alta proporción de ácidos grasos insaturados de cadena larga, (FAO/OMS, 2008) y la presencia de $\beta$-carotenos debido al color naranja intenso del aceite.

Dadas las evidencias encontradas con respecto a la composición nutricional de los parámetros analizados en frutos esféricos y ovalados se extendió el estudio a la determinación de antioxidantes y proteínas en ambos tipos de frutos.

\subsection{SEGUNDA ETAPA}

Se obtuvieron nuevas muestras de 5 plantas con frutos "ovalados", y otras 5 con frutos "esféricos" determinándose en la pulpa seca y molida, proteínas y antioxidantes. Los resultados se describen en la Tabla 1. 


\section{TABLA 1 COMPOSICION NUTRICIONAL DE PULPA DE PINDÓ (SYAGRUS ROMANZOFFIANA) SECA MOLIDA SEGÚN FORMA DE LOS FRUTOS}

\begin{tabular}{lcccc}
\hline \multirow{2}{*}{\multicolumn{1}{c}{ Determinaciones }} & \multicolumn{4}{c}{ Frutos } \\
\cline { 2 - 5 } & \multicolumn{2}{c}{ Ovalados $(\boldsymbol{n}=5)$} & \multicolumn{2}{c}{ Esféricos $(\boldsymbol{n}=5)$} \\
\cline { 2 - 5 } & Media $^{*}$ & $\boldsymbol{\sigma}$ & Media $^{*}$ & $\boldsymbol{\sigma}$ \\
\hline Fenoles totales $(\mathrm{mgEAG/100g)}$ & $6,45^{\mathrm{a}}$ & 0,52 & $7,53^{\mathrm{b}}$ & 0,67 \\
\hline Carotenoides totales $(\mu \mathrm{g} \beta$-caroteno/g) & $160,64^{\mathrm{a}}$ & 59,83 & $213,56^{\mathrm{a}}$ & 73,38 \\
\hline Ácido ascórbico $(\mathrm{mgVit} \mathrm{C} / \mathrm{g})$ & $1,01^{\mathrm{a}}$ & 0,43 & $5,23^{\mathrm{b}}$ & 0,22 \\
\hline Proteínas g/100g de muestra & $3,57^{\mathrm{a}}$ & 0,26 & $3,66^{\mathrm{a}}$ & 0,26 \\
\hline
\end{tabular}

*ANAVA, letras diferentes indican diferencias significativas determinado por Test de Tukey

Los valores de proteínas obtenidos indican que no se observó diferencias significativas con respecto a este componente entre frutos ovalados y esféricos. Los valores hallados se parecen más a los publicados por Goudel (2012), de $4 \mathrm{~g} / 100 \mathrm{~g}$ que a los determinados por Coimbra y Jorge (2012) de 5,41 g/100 g.

Respecto a la vitamina $C$ se encontraron valores con diferencia significativa entre frutos ovalados y esféricos, conteniendo estos últimos mayor concentración, $5,23 \mathrm{mg} / \mathrm{g}$. Los datos de vitamina C en harina de pulpa de pindó determinada por Guimarães (2013), 1,82 mg/g se asemejan más a los obtenidos para pindó ovalados, $1,01 \mathrm{mg} / \mathrm{g}$. La misma autora obtuvo valores de compuestos fenólicos de $1,2 \mathrm{~g}$ de EGA/100 g (GUIMARÃES, 2013), Coimbra y Jorge (2011) publicaron un total de $3,26 \mathrm{~g}$ de EGA/100 g, en pulpa de pindó, ambos valores menores que los hallados en el presente trabajo, en frutos ovalados, $6,45 \mathrm{~g}$ y en esféricos, 7,53 g de EGA/100 g. Éstas diferencias son estadísticamente significativas según la forma de los frutos.

La determinación de carotenoides totales no arrojó diferencia significativa en las concentraciones según la forma, pero fueron superiores a los determinados por Guimarães (2013), $85 \mathrm{ug} / \mathrm{g}$. Coimbra y Jorge (2011), hallaron valores de $1219 \mathrm{ug} / \mathrm{g}$ de carotenoides, pero en aceite de pulpa de pindó, es decir no es válida la comparación puesto que al extraer los aceites, los carotenoides de naturaleza lipídica, se concentran en esta fase.

\section{CONCLUSIONES}

En este trabajo se ha determinado que el contenido de proteínas, y el contenido de carotenoides en la pulpa de frutos de pindó no presenta diferencias significativas en los frutos según la forma.

Se encontraron diferencias significativas en las concentraciones de los compuestos bioactivos, polifenoles y vitamina $C$ en la pulpa de los frutos según su forma, también se evidenciaron diferencias en el perfil de $A G$ y en la proporción de $A G$ saturados/insaturados, en frutos enteros según la forma, presentando en general los frutos esféricos mejor perfil nutricional que los ovalados.

No se encontraron diferencias, según la ubicación geográfica de las plantas. En base a éstos resultados se puede concluir que:

Los frutos son potencialmente aptos para la fabricación de harina de pulpa.

Descartando las pruebas de toxicidad (obligatorias en la evaluación de sustancias alimenticias noveles) los frutos esfericos pueden considerarse aptos para la obtención de aceites comestibles sin necesidad de separar pulpa de semilla. También serían muy beneficiosos como ingrediente de alimentos balanceados.

Los frutos son potencialmente aptos como ingredientes, para el diseño de alimentos funcionales debido a sus componentes bioactivos 


\section{ABSTRAT \\ NUTRITIONAL COMPOSITION FRUITS OF Syagrus romanzoffiana (PINDÓ) NATIVE FROM FORMOSA- ARGENTINA}

The Palm tree "pindó" (Syagrus romanzoffiana) is an abundant native species from province of Formosa, Argentina. The plants produce fruits with different shapes, oval and spherical. The profile of fatty acids (FA) in whole fruits, and antioxidants and proteins in ground dried pulp were measured. It look for answer questions if different fruits formats imply variations in nutritional composition and antioxidant. Experimental data indicates differences in fatty acid profile as a function of the fruit format. spherical fruits showed a relationship between saturated and unsaturated fatty acids, beneficial to human health, in an intermediate profile between the olive and sunflower oils. The protein content of oval fruits was $3.57 \mathrm{~g} / 100 \mathrm{~g}$ and ball was $3.66 \mathrm{~g} / 100 \mathrm{~g}$. Among the antioxidants, vitamin C content showed a significant difference between spherical and oval fruits, the latter containing the highest concentration of $5.23 \mathrm{mg} / \mathrm{g}$. Significant difference was also found in phenolic compounds according to the format of fruit equal to $7.53 \mathrm{mgEAG} / 100 \mathrm{~g}$ for spherical fruits. The concentration of carotenoids was 160.64 to oval format in fruit and $\beta$-carotene $213.56 \mathrm{mg} / \mathrm{g}$ spherical. The AG profile and the proportion of oils found in spherical fruit featuring pindó as suitable source for the extraction of edible oils valuable to health relative to their proportion of saturated fatty acids / unsaturated (1 / $2,5)$ for the fruit full. The high content of bioactive compounds, polyphenols, carotenoids and vitamin C improves the nutritional quality of the fruit pulp and the potential for the use in the enrichment of food.

KEYWORDS: PINDÓ, PALM, NUTRITIONAL COMPOSITION, ANTIOXIDANTS, OIL.

\section{REFERENCIAS}

1 Association of Official Analytical Chemistry (AOAC). Official methods of analysis of AOAC International. 18th ed. Wasington. 2005

2 BONOMO, M ; CAPELETTI ,L. Uso prehispánico de las palmeras Syagrus romanzoffiana y Butia yatay en el Nordeste argentino: aportes desde la etnografía y la biometría Revista del Museo de Antropología v7 n 2.p. 234. 2014

3 BLIGH, E, G ; DYLR, W, J, A. Rapid method of total lipid extraction and purification. Can J. Biochem. Physiol. 911-917. 1957

4 CABRAL, E.; CASTRO, M. Palmeras argentinas. Guía para el reconocimiento. Buenos Aires : L.O.L.A. (LITERATURE OF LATIN AMERICA - LOLA). 2007.

5 COIMBRA, M.C. ; JORGE, N. Proximate composition of guariroba (Syagrus oleracea), jerivá (Syagrus romanzoffiana) and macaúba (Acrocomia aculeata) palm fruits. Food Resarch International. v 44. p. 2139-2142. 2011

6 COIMBRA. M. C.; JORGE, N. Fatty acids and bioactive compounds of the pulps and kernels of Brazilian palm species, guariroba (Syagrus oleraces), jeriva (Syagrus romanzoffiana) and macaúba (Acrocomia aculeata). J Sci Food Agric. v. 92. p. $679-684$. 2012

7 CROCOMO, O.J.; MELO, M. Acrocomia species. En BAJAJ, Y.P.S Biotechnology in Agriculture and Forestry. New Delhi : Springer-Verlag Berlin Heidelberg. 1996.

8 Food and Agriculture Organization of United Nations/ World Helth Organization. FAO/OMS. Fat and fatty acids in human nutrition. Report of an expert consultation. Food and Nutrient. v.98. Roma. 2008

9 GOUDEL F. Caracterização e Processamento de Mapuitã, os frutos da palmeira jerivá (Syagrus romanzoffiana Cham.) 2012. 115 f. Dissertação (Mestrado em Agroecosistemas) - Programa de Pós-Graduação em Agroecossistemas Centro de Ciências Agrárias. Universidade Federal de Santa Catarina, Florianopolis. 2012

10 GUIMARÃES, A. Potencial Funcional e Nutricional de Farinhas de Jerivá (Syagrus romanzoffiana) y Bacaba (Oenocarpus bacaba). 2013. 115 f- Dissertação (Maestrado em Ciencia dos Alimentos). Universidade Federal de Lavras. Lavras, M.G. 2013

11 GUTIÉRREZ AVELLA, D.; ORTIZ GARCÍA, C.; CISNEROS, A. Medición de Fenoles y Actividad Antioxidante en Malezas Usadas para Alimentación Animal. En SIMPOSIO DE METROLOGíA 10. 2008 Santiago de Querétaro- SM2008-M220-1108. 2008. p. $1-5$.

12 LAM, S.; CHEN, J.; KANG, C.; CHEN, C.; LEE, S. $\alpha$-glucosidase inhibitors from the seeds of Syagrus romanzoffiana. Phytochemistry v. 69. p. 1173-1178. 2008

13 NAZARENO; M.; GONZÁLEZ, E.; GARCÍA, M.; LOTO, C.; CORIA CAYUPÁN, Y. Actividad antioxidante de alimentos consumidos en Argentina. Revista de la Asociación Química Argentina: Industria y Quimica v 358. p. 58-63. 2008

14 NDHLALA, A.; MOYO, M.; VAN STADEN, J. Natural Antioxidants: Fascinating or Mythical Biomolecules?. Molecules v.15. p. 6905-6930. 2010

15 PALOMINO G. L. R.; GARCÍA P. C. N.; GIL G. J. H.; ROJANO B. A.; DURANGO, R. D. Determinación del contenido de fenoles y evaluación de la actividad antioxidante de propóleos recolectados en el Departamente de Antioquía (Colombia). Vitae. v.16, n. 3. p. 388-395. 2009

16 PEREIRA DA SILVA B. Y PAZ PARENTE J. Chemical properties and antiinflammatory activity of a galactomannoglucan from Arecastrum romanzoffianum Phytochemistry Letters, v 3. n.3. p.109-112- 2010.

17 RODRIGUEZ AMAYA, D. A guide to carotenoide analysis in food. ILSI Press- USAID. Washington, 2001. $64 \mathrm{p}$

\section{AGRADECIMIENTOS}

Los autores agradecen la colaboración de la Sra. Nilda Rotela, del Sr. Alejandro Field y de la SECyT UNCAus. Este proyecto contó con el apoyo financiero parcial de la SECyT UNaF. 